www.ocula.it • ISSN 1724-7810 • Vol 21, No 24 (October 2020) • DOI: 10.12977/ocula2020-45

Quando è design When is Design Quand c'est du design

a cura di Michela Deni e Dario Mangano

\title{
Soglie e ideologia del progetto
}

\author{
Daniele Barbieri \\ Accademia di Belle Arti di Bologna, IT \\ db@danielebarbieri.it
}

\begin{abstract}
No idea of design exists before William Morris, in the second half of the nineteenth century, came to develop it and put it into practice. Before the Industrial Revolution there were arts and crafts, and certainly there were also designing activities (architects, for example, have been doing it for a long time), but the project in a modern sense, the design, was born from the idea that so-ciety itself can be changed, and predictably, through a project. In his action, Morris designs not only an object, but an activity of social relevance through an object. Before him, objects belonged to the material culture of the time, and were part of it in a traditional way: if they came to trans-form it, it was not an expected consequence.

What should be considered an object is a complex question, however much it can have to be extended to virtual and intangible objects. In any case, a book is an object, even if we make refer-ence only to its verbal or visual content. But a theater play, is it? One of the thresholds that decide when the projectual operation that produces it is design or not, depends on the decision on what is and what is not an object.

Another threshold is more strictly pertinent to the precision with which the social conse-quences of one's action are designed. From this point of view, the domain of art is, for example, completely foreign to that of design, even though there may be objects that fall under both.
\end{abstract}

\section{Keywords}

Design; Semiotics; Object; Project; Ideology. 
Vol 21, No 24 (October 2020) • DOI: 10.12977/ocula2020-45

Daniele Barbieri | Soglie e ideologia del progetto

Accettiamo sensatamente che si attribuisca a William Morris l'invenzione dell'idea di design. Disgustato dalla scarsa qualità estetica degli oggetti prodotti in grande quantità e a basso costo dall'industria, Morris avrebbe sostenuto e diffuso l'idea che la produzione industriale non doveva necessariamente pagare i vantaggi di cui godevano per la prima volta nella storia i suoi prodotti, con una bruttezza che avrebbe imbarbarito inevitabilmente la qualità degli ambienti destinati a ospitarli, e di conseguenza anche la vita quotidiana dei loro utenti. Dimostrò che non era affatto impossibile produrre industrialmente degli oggetti di buona qualità estetica, perché la bellezza degli oggetti influiva sulla qualità della vita dei loro utenti, e se era possibile produrli a prezzi contenuti il miglioramento avrebbe potuto riguardare fasce di popolazione molto ampie, e quindi la società nel suo complesso.

L'idea di design nasce insomma insieme allidea di progetto sociale. Non è certo un caso che siano, quelli, gli stessi anni in cui altre idee di trasformazione razionale della società, basata su un progetto, si stavano facendo avanti, prima tra tutte il marxismo. Pur senza il radicalismo del progetto di Marx, quello di Morris apparteneva alla stessa categoria: era infatti incentrato sul mostrare come fosse possibile progettare, razionalmente, un diverso e migliore modo di vivere.

È questo che distingue, sostanzialmente, il design dal progetto che già esisteva nella tradizione, in particolare quello architettonico. Prima di Morris l'invenzione di un oggetto nuovo non era argomento di una disciplina specifica, e non richiedeva teorizzazioni ideologiche. Poteva accadere, certo, che il progetto non riguardasse solo un oggetto materiale, ma anche una trasformazione sociale legata alla presenza di quell'oggetto: la costruzione delle cattedrali, in Europa, è sempre stato qualcosa di questo genere. In particolare, se vogliamo passare dall'azione corale e condivisa (anche quando al suo centro stava comunque un architetto di genio) che caratterizzava la costruzione delle cattedrali gotiche, a qualcosa di più modernamente progettato secondo un criterio sufficientemente esplicito e chiaro, il caso del Cupolone di Santa Maria del Fiore a Firenze può essere visto come esemplare. Progetto razionale architettonico quello di Ser Pippo, ma - ancora più interessante - progetto razionale politico quello di Giovanni de Medici che, finanziando quest'opera ai limiti delle possibilità umane, ridava entusiasmo e coesione alla comunità fiorentina, ponendo inoltre solidissime basi al futuro potere della città sulla Toscana e della propria famiglia nella città stessa.

Ma né il Medici né Brunelleschi avevano in mente un processo globale, né alcuna utopia di riferimento. La pretesa di origine illuminista e positivista di poter controllare razionalmente il mondo sociale e la sua trasformazione ha la sua origine e il suo sviluppo nel corso dell'Ottocento. Nel Novecento arriva in diversi luoghi del mondo a gestire la politica di grandi paesi al massimo livello, con conseguenze più volte tragiche: se si ritiene che il benessere collettivo possa essere raggiunto attraverso la realizzazione di un'idea (sia essa il comunismo, la purezza della razza o anche solo l'omogeneità etnica di una nazione), qualsiasi strage viene giustificata dal progetto razionale. Non è in gioco nessuno scatenamento irrazionale, ma solo il perseguimento coerente 
Vol 21, No 24 (October 2020) • DOI: 10.12977/ocula2020-45

Daniele Barbieri | Soglie e ideologia del progetto

di un progetto sociale, nello sterminio sovietico per carestie programmate dei Kulaki, in quello nazista degli Ebrei o in quello turco degli Armeni. Il sogno della ragione può generare mostri, assai più del suo sonno (e che la parola spagnola sueño possa essere tradotta in ambedue i modi getta una luce diversa sul senso di quell'acquaforte di Goya - il medesimo pittore che dipingeva anche i fucilati spagnoli dagli invasori francesi, che morivano gridando "Abbasso la ragione!”).

Ben difficilmente il design come normalmente lo intendiamo potrà arrivare a conseguenze come queste, nemmeno quando lo chiamiamo social design, rendendo esplicita una vocazione di trasformazione sociale su base ideale, anziché una di conservazione o di trasformazione su base commerciale. Benché il designer si debba porre il problema di quale tipo di società venga favorita dal proprio progetto, sarà in tutti i casi consapevole che la portata della propria azione rimane molto più limitata. Tuttavia, fatta salva questa differenza di formato, la questione di fondo rimane comune, e le conseguenze sociali del proprio progetto ne fanno a tutti gli effetti parte, almeno nella misura in cui possono essere previste.

Il Sein und Zeit, Martin Heidegger (1927) individua nel progettare (entwerfen) il nocciolo della nostra condizione esistenziale, e nell'essere gettati (Geworfenheit) la sua natura. In tedesco le due espressioni sono ancora più legate etimologicamente tra loro di quanto lo siano in italiano: il progetto è l'inevitabile risposta all'essere gettati nel mondo (un pro-ietto che si contrappone a un de-ietto). Certo, l'idea di design si ritrova a sua volta promossa da questa posizione, ma c'è in essa qualcosa di più che nell'entwerfen heideggeriano, il quale consiste sostanzialmente in una prospettività dell'azione umana, volta al futuro. Per questo è necessario distinguere il più precisamente possibile il campo specifico del design da quello di altre forme di progetto, individuando delle soglie, che separino quando è design da quando non lo è.

Per esempio, la natura di progetto razionale del design lo distingue in linea di principio dall'arte: in linea di massima, quando è design non è arte, e viceversa. Non lo era nemmeno nel passato, quando quel fenomeno sostanzialmente rituale che è l'arte celebrava i miti del proprio presente - e, da questo punto di vista, pure il Cupolone è una celebrazione rituale. Progetto razionale quello di ser Pippo, progetto razionale quello di Giovanni de Medici, ma per giungere a una celebrazione rituale della città e dei suoi cittadini. Valutando quell'operazione oggi, ci terremmo probabilmente a sottolineare le componenti civiche (e quindi sociali) del progetto di entrambi; ma prima che Morris ci inducesse a pensare in questo modo, la componente estetica, e quindi rituale, celebrativa, avrebbe riempito senza remore le nostre considerazioni.

Nell'epoca in cui il design è nato davvero, la differenza tra arte e design si è indubbiamente polarizzata. Con la complicità (già preromantica) delle poetiche del sublime, l'arte si è tendenzialmente allontanata da una dimensione mitica di carattere sociale (non senza remore, e produttivi riavvicinamenti). In fin dei conti, il massimo valore che l'arte può celebrare da fine Ottocento in poi (l'epoca in cui la trasformazione è effettivamente arrivata al suo compimento) è il dubbio razionale, la messa in discussione o in crisi. Se il progetto del desi- 
Vol 21, No 24 (October 2020) • DOI: 10.12977/ocula2020-45

Daniele Barbieri | Soglie e ideologia del progetto

gn è un progetto in positivo (si progetta una forma di vita a venire, un dettaglio sociale da realizzare o perfezionare), quello dell'arte è invece un progetto in negativo (si progetta la messa in discussione di una forma di vita, il dubbio, magari produttivo). Che il dubbio possa rivelarsi produttivo spiega perché i confini tra design e arte non siano affatto netti, e rimanga possibile un'ampia zona di sovrapposizione. Resta il fatto che i reciproci ambiti sono comunque differenti: all'arte, per esempio, non si richiede affatto di preoccuparsi delle proprie conseguenze; al design invece certamente sì.

Non è design, d'altra parte, nemmeno quando il progetto non c'è, cioè quando si arriva per caso, o per ispirazione del momento all'oggetto, al risultato anche brillante. Non è design, insomma, l'improvvisazione, una pratica che può invece essere anche cruciale per l'arte. Questo non vuol dire che un designer non possa avere una brillante e improvvisa intuizione sulla fattura di un oggetto: vuol dire semmai che l'intuizione di un momento deve poi essere valutata e soppesata sulla base delle sue possibili conseguenze. Solo in questo senso sarà progetto razionale. Alle indimenticabili improvvisazioni jazzistiche di John Coltrane non avrebbe nessun senso fare la medesima richiesta.

Meno basilare mi sembra la presenza o assenza dell'oggetto materiale. Parliamo più facilmente di design quando il prodotto è un oggetto, mentre parlare del design di una performance teatrale, o di una manifestazione politica ci sembra più una metafora che un uso legittimo. Ma qui credo che giochi la componente disegno del termine design. Se parlassimo del progetto razionale di una manifestazione politica, credo che nessuno avrebbe da ridire. Anche l'uso di disegno nel senso di progetto, per quanto accettato e assunto dai dizionari come letterale, ci suona vagamente metaforico.

Non si può trascurare il fatto che una parte consistente del design riguarda oggetti che sono comunque oggetti cognitivi: il graphic design o design della comunicazione produce oggetti che sono testi. Da questo punto di vista, la scrittura di testi è un atto di design (implicito, ma forse non tanto quanto quello del design propriamente detto) molto antico, perché un testo è comunque il prodotto di un progetto razionale che mira a dare una certa forma al mondo (confermandolo come è o cercando di cambiarlo, come anche da Platone in poi). Un testo è uno strumento, proprio come un cacciavite.

Qualcuno obietterà magari che un cacciavite produce alterazioni pratiche del mondo (viti avvitate) mentre un libro produce alterazioni cognitive (menti consapevoli). Ma è davvero pertinente questa distinzione? Non testimonierà piuttosto un forte debito nei confronti dell'opposizione cartesiana tra res extensa e res cogitans, magari nella forma rimodernata di mondo vs mente? E anche volendo restare nei termini tradizionali, se un prodotto di design deve trasformare (per quanto magari in minima misura) come potrebbe non produrre anche alterazioni cognitive?

In una prospettiva un po' da ecologia della mente ${ }^{1}$, potremmo provare a considerare in generale il vivente come qualcosa che, prima di tutto, mira a ridurre la complessità del mondo circostante. Un essere vivente primitivo ed elementare, ancora senza organi di movimento e senza sensi, viene attraversa-

1 Cioè quella che si trova impostata in Bateson (1972). 
Vol 21, No 24 (October 2020) • DOI: 10.12977/ocula2020-45

Daniele Barbieri | Soglie e ideologia del progetto

to da quello che lo circonda, trattenendo ciò che gli è utile trattenere. Eppure già questa scelta di base è un proto-senso, qualcosa che categorizza il mondo secondo almeno due aree distinte: ciò che è assimilabile e ciò che non lo è. Se questa scelta venisse fatta da organi sensibili esterni, si eviterebbero molte delle sgradevoli conseguenze dell'essere attraversati, per esempio, da un veleno. Se l'organismo possedesse anche organi di movimento, essi lo porterebbero tendenzialmente verso ciò che è assimilabile, allontanandolo tendenzialmente da ciò che è pericoloso. Ogni espansione di sensibilità categorizza più finemente il mondo. Ogni espansione di attività favorisce ulteriori espansioni di sensibilità.

A un livello di complessità molto maggiore, scegliersi o, ancor di più, costruirsi una tana riduce fortemente la complessità del mondo, ora facilmente categorizzabile sulla base dell'opposizione tana vs non-tana, ovvero luogo del riposo e della sicurezza, contrapposto al luogo dell'azione (anche nutritiva) e del pericolo. La tana dell'animale è forse il proto-strumento, la prima modificazione dell'ambiente che ne permette una qualche forma di controllo, e quindi di semplificazione, di riduzione della complessità.

Nella misura in cui lo strumento divide il mondo in quello su cui esso stesso può operare e quello che no, ecco che lo strumento ci fornisce una prospettiva che è al tempo stesso operativa e cognitiva: la riduzione di complessità farà sì che io possa operare con maggiore sicurezza, e lo strumento ne sarà il tramite. Nel momento in cui nasce il segno simbolico, esso si rivela uno strumento molto potente: poter fare riferimento a qualcosa che non è presente (non solo cose, ma anche un'azione futura) comporta una facilità di azione combinata tra gli eventuali diversi attori in gioco, ma comporta anche la possibilità di una memorizzazione non soltanto operativa. Per un vivente che abbia una memoria esclusivamente operativa (sappia cioè imparare a fare, ma niente più) l'insieme delle condizioni esterne (naturali o costruite che siano) è fondamentale. Ma una memoria simbolica è in grado di costruire comunicativamente dei sostituti del mondo che lo sono in misura sufficiente perché si possa parlare di progetto nel senso ampio di Heidegger.

La parola è dunque uno strumento operativo cruciale, ancora prima che comunicativo. Il mito omerico dà forma alla società greca antica, diffondendone e rinsaldandone le basi sociali e culturali, dando un fondamento alle gerarchie, ai costumi e persino ai dettagli tecnici nella costruzione delle navi. Non è certo un progetto razionale, e non è certo design in questo senso. Ma quando Platone, qualche secolo dopo, in un contesto assai più direttamente e maturamente politico, descrive per iscritto la propria Repubblica, la sua scrittura è comunque un tentativo razionale (operativamente fallimentare alla breve, ma fertilissimo alla lunga) di trasformare il mondo per mezzo di sequenze organizzate di parole. In questo senso, potremmo considerare l'operazione platonica come l'antenato diretto dell'invenzione di Morris.

Il progetto razionale mira a controllare il mondo, riducendone la complessità, come fa peraltro qualsiasi strumento. In questo senso, un cacciavite è uno strumento esattamente come una teoria contenuta in un libro. Evidentemente il livello di complessità intrinseca a questi diversi strumenti è molto diver- 
Vol 21, No 24 (October 2020) • DOI: 10.12977/ocula2020-45

Daniele Barbieri | Soglie e ideologia del progetto

so, come è diverso l'impatto trasformativo implicito nel progetto. Le parole sono strumenti che servono per costruire altri strumenti (tra cui parole stesse) che servono per costruire altri strumenti, con un impatto culturale-sociale potenzialmente significativo; e quando dico "parole" non mi riferisco solo alle sequenze fonetiche o grafiche, ma a tutto il complesso di regole che tiene insieme il linguaggio verbale. Il cacciavite non è adatto né a costruire altri cacciaviti, né ad articolarsi troppo con i propri parenti strumentali. D'altra parte, nessuna sequenza di parole sarà mai in grado di avvitare una vite - anche se potrà avviare un processo a cui prenda parte un cacciavite che avviterà una vite. Il design di Morris possiede implicitamente un carattere più vicino, in questi termini, a quello del libro piuttosto che a quello del cacciavite.

Jean-Marie Floch ci proponeva ${ }^{2}$ qualche anno fa di analizzare il suo coltellino Opinel come un soggetto manipolato, cui si fa fare questo e quello. Cosa succederebbe se trattassimo in questo modo anche un testo verbale? Quali sono le affordances della Critica della Ragion Pura, che ci permettono di farla operare in qualche modo nel nostro discorso? Quali sono le viti che quel tipo di cacciavite è in grado di avvitare? Io non dubito affatto che i classici strumenti (!) narratologici della semiotica generativa ${ }^{3}$ ci possano essere di grande utilità in questo senso, ma qui è proprio il senso di interpretazione che cambia: trattare un testo verbale come uno strumento significa interpretarlo nei termini della sua operatività. Il suo significato, alla Wittgenstein, ${ }^{4}$ starebbe in ciò che fa, o che può fare. Ridurlo alle opposizioni semiche fondamentali vorrebbe dire individuare quali ne siano le componenti massimamente operative, e come possano agire sul mondo. $\mathrm{E}$ in questo modo, magari, intuire anche come si possano costruire strumenti verbali con caratteristiche simili per ottenere efficacie simili, o anche superiori. La semiotica è inevitabilmente una teoria delle teorie, apoteosi delle possibilità intrinseche stesse dello strumento segnico e in particolare linguistico.

Ora, proviamo a valutare, data una vite, come essa venga interpretata diversamente per mezzo di un cacciavite, di un martello o di un cucchiaio, tre strumenti che ne valorizzano tre insiemi di proprietà diversi, e che rendono strumenti diversi la medesima vite: qualcosa che può essere fatta penetrare nel legno per avvitamento, qualcosa che può essere fatta penetrare nel legno per percussione violenta e ripetuta, qualcosa che può essere raccolto e sollevato. Guardare il nostro rapporto con il mondo per mezzo della Critica della Ragion Pura, della Fenomenologia dello Spirito o della Gaia Scienza cambia ampiamente il modo di vedere strumentalmente noi stessi. Che cosa succede se lo strumento è Edipo re? O se è Ossi di seppia?

Anche se il progetto razionale esiste certamente da Platone in poi, solo in ambito filosofico è stato teorizzato, e nemmeno troppo accuratamente. Il precetto marxiano secondo il quale sarebbe ora che i filosofi smettano di limitar-

2 Per esempio, in Floch (1995).

3 Il riferimento è ovviamente Greimas (1983), con tutta la letteratura che ne segue.

4 Il Wittgenstein del 1953, evidentemente. 
Vol 21, No 24 (October 2020) • DOI: 10.12977/ocula2020-45

Daniele Barbieri | Soglie e ideologia del progetto

si a interpretare il mondo, cercando piuttosto di trasformarlo (magari, certo, sulla base di quella stessa interpretazione) si ritrova nell'invenzione morrissiana del design. Gli oggetti, forse per la prima volta nella storia, vengono eletti a operatori razionali di trasformazione, sul modello della parola. È design quando si agisce in questo modo, all'interno di un'ideologia del design che ha segnato gli ultimi due secoli insieme ad altre ideologie, non sempre ugualmente proficue.

\section{Bibliografia}

Bateson, Gregory

1972 Steps to an Ecology of Mind, Chandler P.C. (tr.it. Verso un'ecologia della mente, Milano, Adelphi, 1976).

Greimas, Algirdas Julien

1983 Du sens II. Essais sémiotiques, Paris, Seuil (tr. it. Del senso II. Narrativa, modalità, passioni, Milano, Bompiani, 1985).

Heidegger, Martin

1927 Sein und Zeit, Tübingen, Max Niemayer Verlag (tr. it. Essere e tempo, Milano, Longanesi, 1976).

Floch, Jean-Marie

1995 "Il fondamento antropologico del design. Il caso del coltellino Opinel", in Pozzato, Maria Pia (a cura di), Estetica e vita quotidiana, Milano, Lupetti.

Wittgenstein, Ludwig

1953 Philosophische Untersuchungen, Oxford, Basil Blackwell (tr. it. Ricerche filosofiche, Torino, Einaudi, 1974).

Daniele Barbieri semiologo, si occupa di comunicazione visiva, ma anche di poesia e di musica. Insegna presso l'Accademia di Belle Arti di Bologna, l'ISIA di Urbino, l'Università di San Marino.

Tra i libri pubblicati: I linguaggi del fumetto (Bompiani 1991), Questioni di ritmo (ERI-RAI 1996), Nel corso del testo. Una teoria della tensione e del ritmo (Bompiani 2004), Breve storia della letteratura a fumetti (Carocci 2009), Guardare e leggere. La comunicazione visiva dalla pittura alla tipografia (Carocci 2011), Il linguaggio della poesia (Bompiani 2011), Semiotica del fumetto (Carocci 2017), Letteratura a fumetti? Le impreviste avventure del racconto (ComicOut 2019), Testo e processo. Pratica di analisi e teoria di una semiotica processuale (Esculapio 2020).

On line: <http://www.guardareleggere.net>. 